\title{
Low-Energy Sonic Thermographic Inspection of Impact Damage in Aerospace Composites
}

\author{
Kelly A. Tsoia ${ }^{a *}$, Chris Brooks ${ }^{b}$, Nik Rajic ${ }^{c}$ and Cedric Antolis ${ }^{d}$ \\ Defence Science and Technology Group, 506 Lorimer Street, Fishermans Bend 3207, Australia \\ aKelly.Tsoi@dst.defence.gov.au, bChristopher.Brooks@dst.defence.gov.au \\ cNik.Rajic@dst.defence.gov.au, ds3448276@student.rmit.edu.au
}

(C) 2020 Commonwealth of Australia

Keywords: Sonic Thermography, Non-Destructive Testing, Low Energy, Kissing Bonds

\begin{abstract}
This paper details an investigation into the use of an alternate sonic thermography inspection process which uses both low-energy acoustic coupling and an inexpensive microbolometer camera to detect barely visible impact damage in composite structures. The impetus is to create a system that is affordable, robust, rugged and easy to use in the field. This paper shows that the new system is capable of detecting barely visible impact damage with a performance comparable to that of conventional sonic thermography.
\end{abstract}

\section{Introduction}

The transformation of the Australian Defence Force (ADF) aviation fleet has seen a significant investment in next generation aircraft platforms, bringing cutting-edge war fighting capabilities to the ADF. Many of these platforms contain significant quantities of composite materials, e.g. F-35A, MRH90 and Tiger ARH. The many advantages of composite materials over metals for structural applications have to be balanced against some shortcomings, such as increased susceptibility to impact damage, especially barely visible impact damage (BVID), as well as kissing bond delaminations. These damage modes are particularly insidious, as they are difficult to detect with conventional non-destructive inspection (NDI) techniques and can cause significant reductions in the static and fatigue strength of components.

Sonic thermography (ST) is an NDI technique that has proven to be effective on a large variety of composite defects and flaws such as BVID, kissing bonds and delaminations [1]-[2]. ST relies on the use of an acoustic horn, typically operating in the frequency range $20-40 \mathrm{kHz}$, to transfer acoustic waves to the structure under inspection. These acoustic waves pervade the structure and interact with the faying surfaces of damaged zones, causing frictional heating. When this heat diffuses to the surface it can be detected using an infrared camera (Fig. 1). The technique works well in the laboratory, however transitioning the technology to the field is challenging. Traditionally, cooled infrared (IR) cameras are used for such inspections, due to their relatively high temperature sensitivity. However, IR cameras of this type are expensive, fragile and bulky to use and transport. In addition, the effective transfer of high frequency acoustic waves from an acoustic horn is reliant on the entire contact area of the horn tip sitting flush against the structure under inspection. For structures that are not completely flat, as is common for many aircraft components, insufficient energy may be transferred resulting in reduced frictional heating and therefore reduced ability to detect defects and delaminations.

A technique has been developed, and is described here, that uses only $5 \%$ of the acoustic power typically applied in traditional ST. This alternative form of ST, referred to here as LowAmplitude Synchronous Sonic Thermography (LASST), uses a cross-correlation technique

(c) (1) Content from this work may be used under the terms of the Creative Commons Attribution 3.0 license. Any further distribution of this work must maintain attribution to the author(s) and the title of the work, journal citation and DOI. Published under license by Materials Research Forum LLC. 
previously implemented in a thermoelastic stress analysis (TSA) approach described in [3]. TSA is based on the thermoelastic effect wherein an object under elastic deformation produces small reversible changes in temperature. These reversible temperature changes can be detected when the object under investigation is mechanically cycled at a known frequency. Knowledge of this frequency is used to extract the relatively small linearly-correlated signature from thermal imagery obtained from a low-cost microbolometer.

Analogously, LASST uses an acoustic horn which is cycled on and off at a known frequency, with the thermal signature generated by frictional heating at flaw surfaces synchronously averaged with respect to the known modulation frequency. Earlier researchers explored the use of a similar process, which has been variously described as amplitude modulated lock-in vibrothermography [4], ultrasound lock-in thermography [5] and ultrasound burst phase thermography [6], however these implementations all used high power ultrasound (from $300 \mathrm{~W}$ up to $1.6 \mathrm{~kW}$ ) and a cooled focal plane array infrared camera. In LASST, the power levels required from the horn are lower by a factor of 20 compared to standard ST and a lightweight, low-cost microbolometer is used. The present paper describes an experimental comparison of LASST against conventional ST and phased array ultrasonic testing (PAUT) on composite laminates containing BVID.

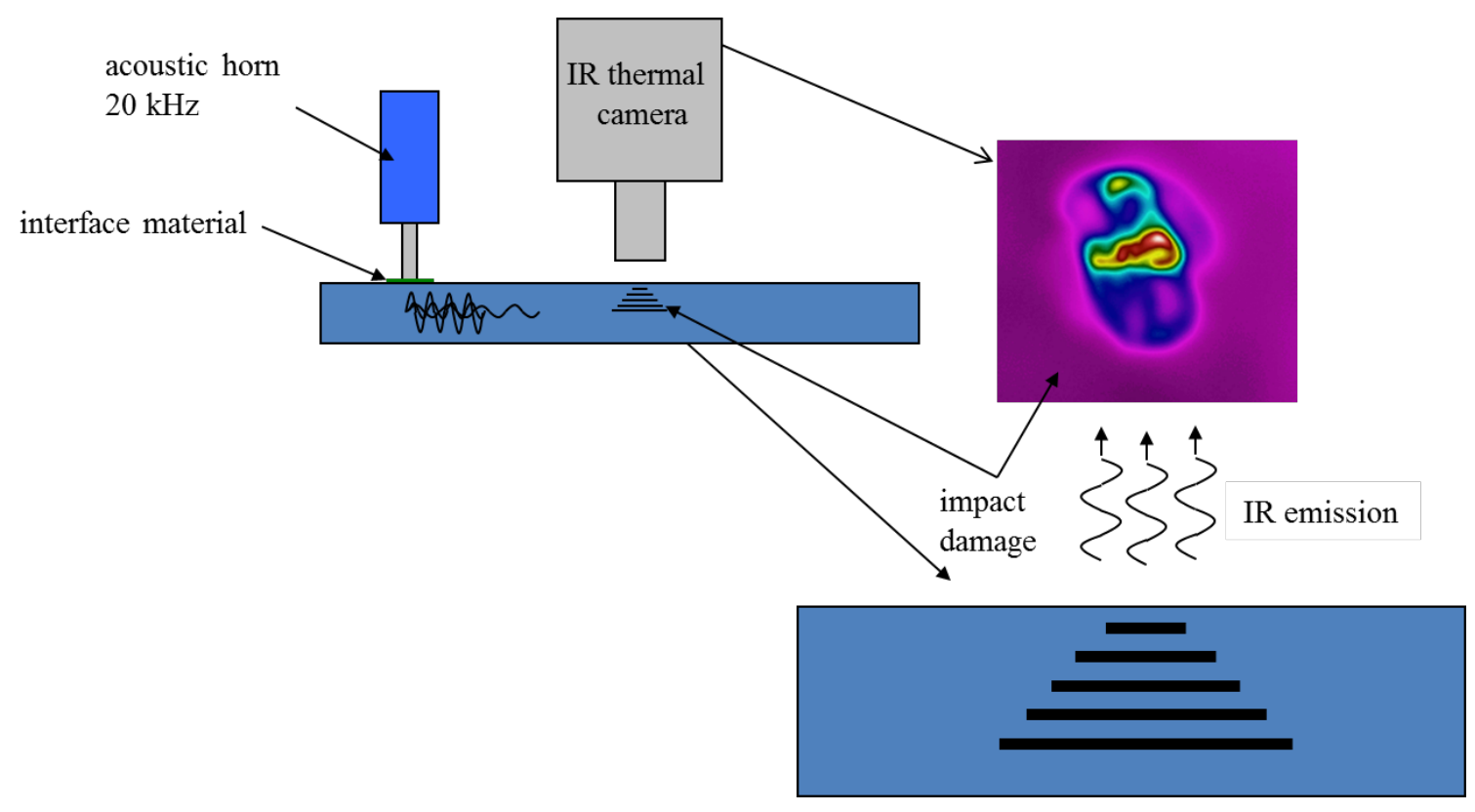

Figure 1. Schematic showing basic operation of ST inspection.

\section{Experiment}

A series of quasi isotropic IM7/977-3 [45,0,0,-45,90]3S laminate plate coupons measuring $300 \mathrm{~mm} \times 100 \mathrm{~mm} \times 4 \mathrm{~mm}$ were impacted at three energy levels within the BVID regime; at $5.36 \mathrm{~J}, 10.72 \mathrm{~J}$ and $21.44 \mathrm{~J}$. The size and lateral extent of the damage was determined using PAUT.

In traditional implementations of ST the acoustic horn is typically driven at power levels of 100 's of watts over durations in the order of $1 \mathrm{~s}$ [1]. An interface material of felt is used to improve energy transfer from the horn into the specimen, and to prevent possible marring of the surface from the horn. As previously remarked, the resulting thermal signature is typically 
recorded using a cryogenically-cooled photon detector. Such cameras have a relatively high sensitivity, with noise equivalent temperature difference (NETD) values typically $<20 \mathrm{mK}$, but also are relatively large, with dimensions of approximately $200 \mathrm{~mm}$ x $150 \mathrm{~mm}$ x $150 \mathrm{~mm}$ and weigh many kilograms. Although the sensitivity of these cameras is relatively high, post processing techniques are still typically applied to the raw thermographic data to improve the probability of detecting a defect; these techniques include pulsed phase thermography (PPT) [7] and principal component thermography (PCT) [8].

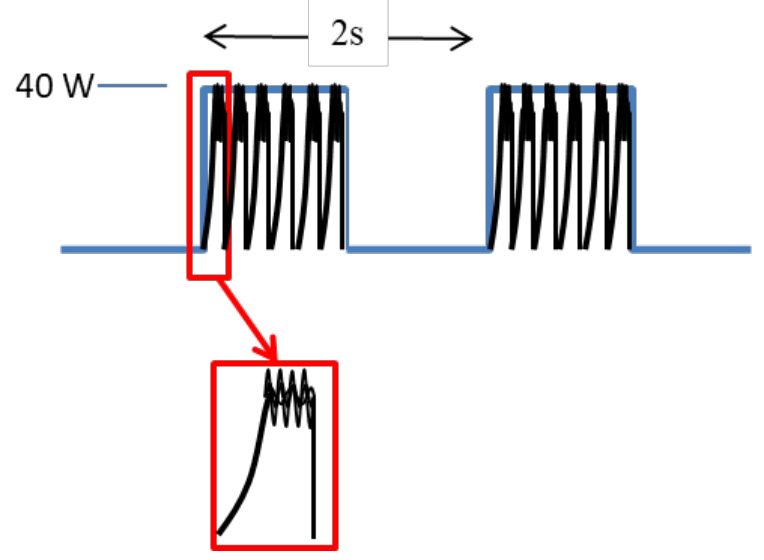

\section{Figure 2. Insonification waveform used in LASST.}

In the present implementation of LASST, pulsed insonification is required as the ultrasonic generator is unable to supply a steady output at low power. Sonic insonification pulses were generated at a frequency of $6 \mathrm{~Hz}$, with each insonification ramping up from zero to the chosen energy level within $0.15 \mathrm{~s}$. The acoustic horn power was modulated using a square wave with a base frequency of $0.5 \mathrm{~Hz}$, which gives $1 \mathrm{~s}$ of pulsed insonification and $1 \mathrm{~s}$ of zero insonification. Each complete $2 \mathrm{~s}$ pulse is considered one cycle, and we collect 20 cycles, which is defined as one block, so each block takes $40 \mathrm{~s}$ to complete. The thermal signature is synchronously averaged against the base frequency of the square wave. The low magnitude of the base frequency is important to enable enough time for the thermal signature to diffuse from the substructure to the surface where it can be detected.

Two infrared imaging systems were used in the present study; (i) a FLIR SC6000 cooled photon detector operating in the 2-5 micron wavelength band with an NETD of $20 \mathrm{mK}$, and (ii) a Xenics Gobi 640 amorphous-silicon microbolometer operating in the 8-12 micron wavelength band and with an NETD of $50 \mathrm{mK}$. The comparatively small size of the microbolometer is illustrated in Fig. 2.

Due to the relatively low intensity and short duration power bursts required by the LASST approach, a thinner interface layer than is customarily applied in ST was able to be used in this work. A thin layer of cardboard, similar to a business card, was found to be sufficient to enable good energy transfer whilst also protecting the surface.

The cross-correlation process applied to the raw video signal in LASST results in two measured signal components: a component in phase with the excitation, which is denoted by the symbol $\mathrm{X}$, and a component in quadrature with the excitation, which is denoted by the symbol $\mathrm{Y}$. Corresponding amplitude $(r)$ and phase $(\Theta)$ components can be calculated from these measured components. Unfortunately, because of unknown latencies in the data acquisition system the precise phase relationship between the thermal response and the acoustic excitation was not able 
to be determined. An empirical phase calibration was instead applied by assigning the relative phase between the $\mathrm{X}$ component and the drive signal at the centre of the impact zone to zero. The phase component relates to the time it takes for the heat to diffuse to the surface, which should approach zero at the centre of the impact zone because any surface, or near-surface damage will produce an approximately instantaneous thermal response to the insonification. This phase calibration process ensures that any unknown or variable latencies in the data acquisition system do not produce inconsistencies between different sets of results. Fig. 4 shows the effect of this calibration process on $\mathrm{X}$ and $\mathrm{Y}$ components obtained from a typical inspection of BVID. As expected, the amplitude component, $r$, is unchanged by a shift in phase.

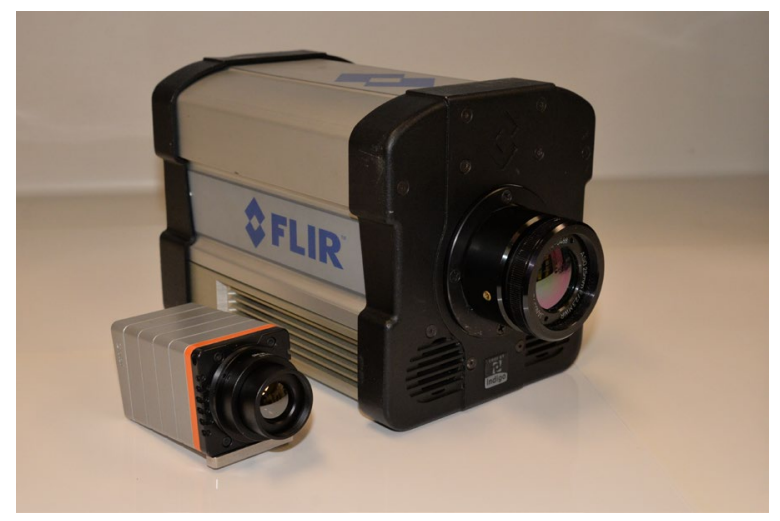

Figure 3. Xenics Gobi microbolometer (left) and FLIR SC6000 photon detector (right).

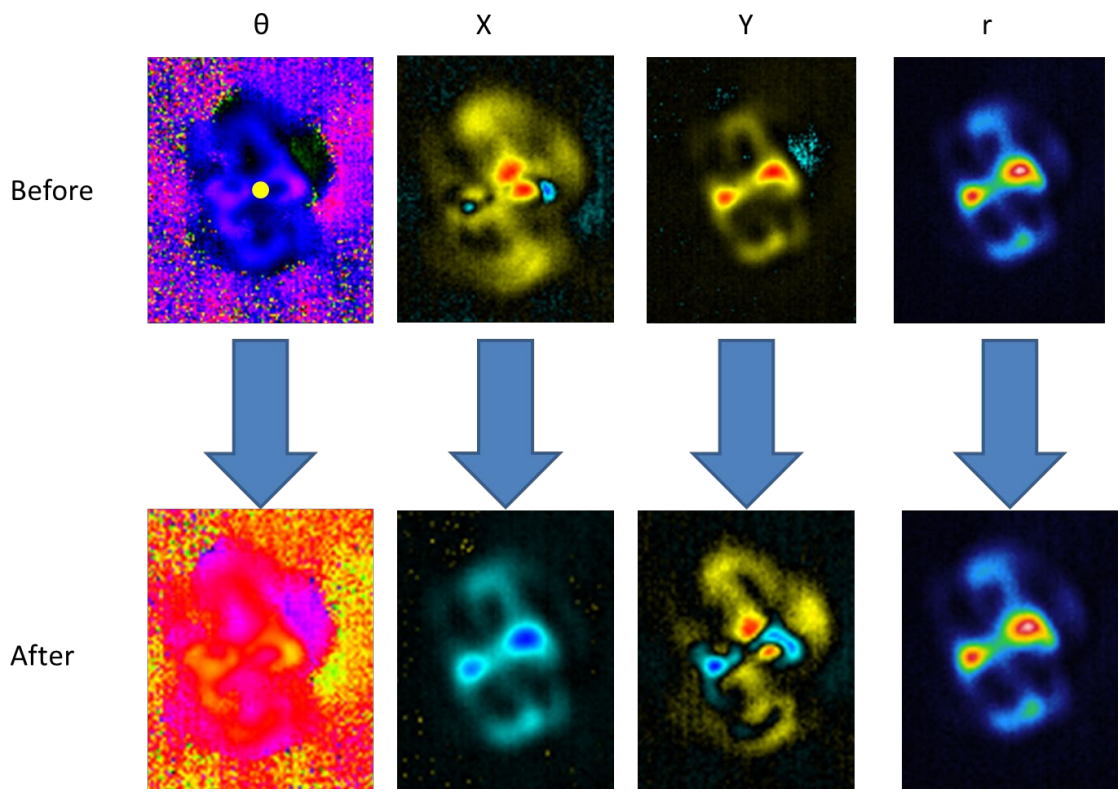

Figure 4. Effect of phase calibration on signal components obtained for $21.44 \mathrm{~J}$ impact specimen. The yellow spot at the centre of $\Theta$ indicates the position where the calibration was performed.

In Fig. 5, the sub-surface damage area at a depth range $0.8-1.2 \mathrm{~mm}$, determined from PAUT, has been outlined in red, and deeper damage, in the depth range 1.2-2.4 mm, in yellow. These 
have been overlaid on the LASST X and Y components to illustrate the good correlation between the $\mathrm{X}$ component signature and near surface damage, and between the $\mathrm{Y}$ component signature and deeper damage. This is to be expected as the $\mathrm{Y}$ component is in quadrature with the horn excitation and therefore corresponds to a delayed response such as would occur for a signal transported from sub-surface layers by heat diffusion.

A comparison of signal strength with energy level was also conducted. It was observed that $30 \mathrm{~W}$ was the minimum power level required to elicit a detectible signature in the BVID samples considered here. A comparison between the $30 \mathrm{~W}$ and $40 \mathrm{~W}$ scans is shown in Fig. 6 for a $21.44 \mathrm{~J}$ impact specimen, where the $40 \mathrm{~W}$ scan exhibits significantly less noise and better signal definition. Based on this result, $40 \mathrm{~W}$ was the minimum energy level applied in all subsequent inspections examining this particular type of damage.

(a)

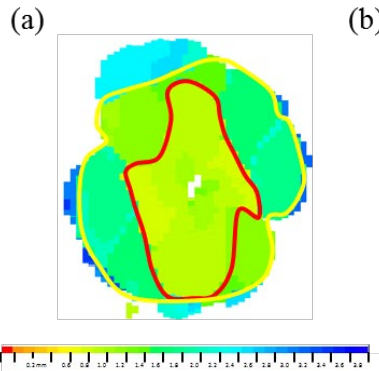

(b)

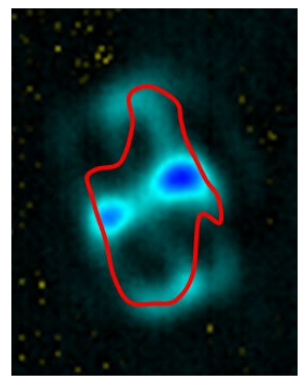

(c)

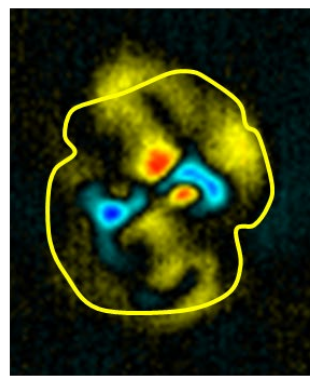

Figure 5. Comparison of (a) PAUT, (b) X component and (c) Y component from LASST for $21.44 \mathrm{~J}$ impact specimen, $40 \mathrm{~W}, 800 \mathrm{~s}$. Depth range $0.8-1.2 \mathrm{~mm}$ is outlined in red and $1.2-2.4 \mathrm{~mm}$ in yellow.
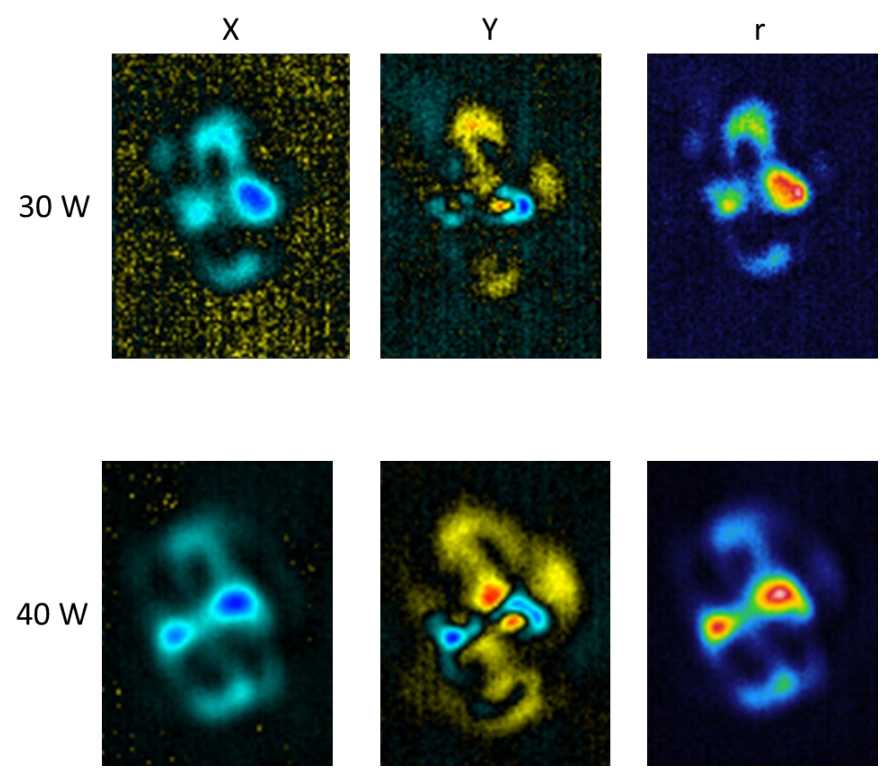

Figure 6 Effect of insonification energy level for 21.44 J impact specimen. Shown are the $X, Y$ and components for $30 \mathrm{~W}$ (above) and $40 \mathrm{~W}$ (below). 
The duration of the scans was varied from $80 \mathrm{~s}$ to $800 \mathrm{~s}$ to determine the minimum length of time during which LASST should be performed to optimise the thermal signature, using a $40 \mathrm{~W}$ insonification power level. A comparison of the results for increasing scan duration is shown for $\mathrm{X}$ and $\mathrm{Y}$ components in Fig. 7. An easily distinguished BVID signature is evident in both $\mathrm{X}$ and $\mathrm{Y}$ components at $80 \mathrm{~s}$, however from $320 \mathrm{~s}$ onwards the damage indication becomes more refined and specific areas of damage are more easily delineated, which is an expected result of the cross-correlation process. For the remainder of the experiments a scan duration of $800 \mathrm{~s}$ was used to ensure that the most defined thermal response was obtained.

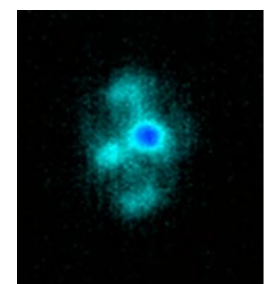

$80 \mathrm{~s}$

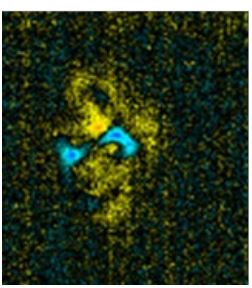

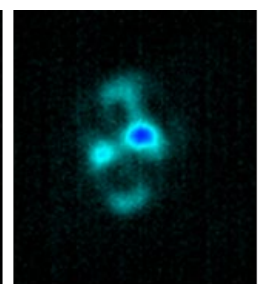

$320 \mathrm{~s}$

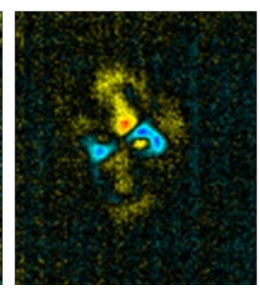

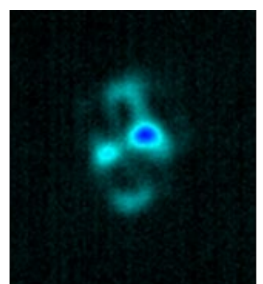

$760 \mathrm{~s}$

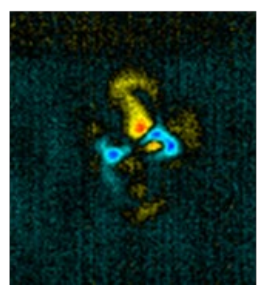

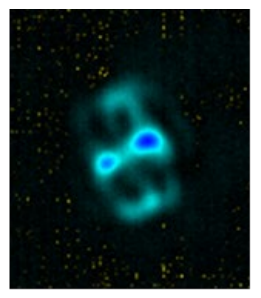

$800 \mathrm{~s}$

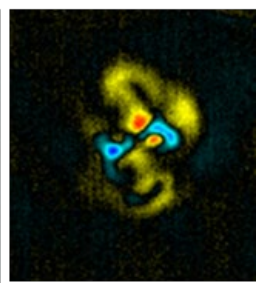

Figure 7. Comparison of component $X$ (above) and $Y$ (below) for LASST thermographs of $B V I D$ in $21.44 J$ impact specimen, over increasing duration of scans at $40 \mathrm{~W}$ insonification.

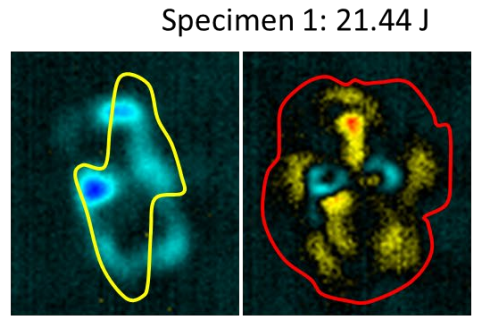

Specimen 4: 10.72J

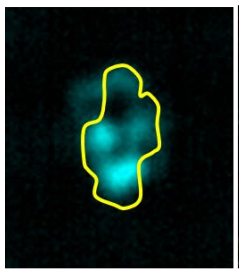

Specimen 2: $21.44 \mathrm{~J}$

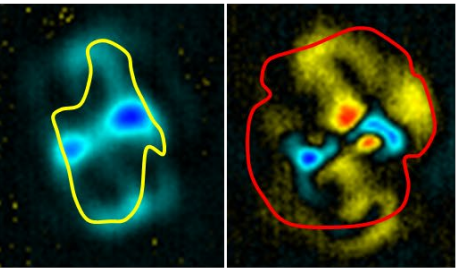

Specimen 5: 10.72J

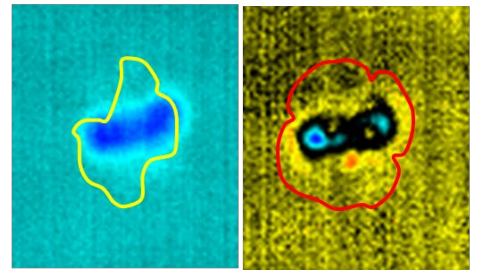

Specimen 3: 21.44J

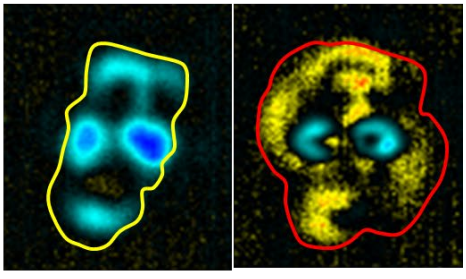

Specimen 6: 5.36J
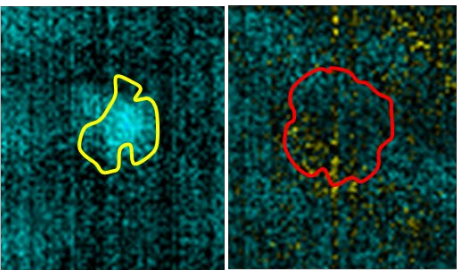

Figure 8. Overlay of damage area obtained from PAUT corresponding to depth ranges of 0.2-1.2 mm (yellow) and 1.2-2.2 $\mathrm{mm}$ (red), onto the $X$ and $Y$ components for each specimen; the impact energies are also listed.

Fig. 8 shows the $\mathrm{X}$ and $\mathrm{Y}$ components for each impact specimen; as before, the yellow outline corresponds to the damage area in the depth range $0.2-1.2 \mathrm{~mm}$ and the red outline to the damage area in the depth range $1.2-1.8 \mathrm{~mm}$, both obtained from the PAUT scans. As indicated 
previously, the $\mathrm{X}$ component should relate most strongly to near-surface damage and the $\mathrm{Y}$ component to deeper damage, and to the extent that the $\mathrm{Y}$ signature is consistently larger in area than the $\mathrm{X}$ signature, they do. An exception is the $5.36 \mathrm{~J}$ impact case, for which a $\mathrm{Y}$ component signature could not be detected.

Fig. 9 shows a comparison between results obtained from a $21.44 \mathrm{~J}$ specimen using the photon detector and the microbolometer. The former provides a more detailed signature of the damage when compared with the microbolometer results, however the main features of the defects are discernible in both. The photon detector result also contains evidence of vibration modes, or standing waves, established in the specimen by the acoustic excitation, which are not present in the microbolometer result, presumably due to the lower sensitivity of that camera. This could be viewed as a practical advantage to using a microbolometer, as these vibrational signatures have the potential to obscure indications of damage.

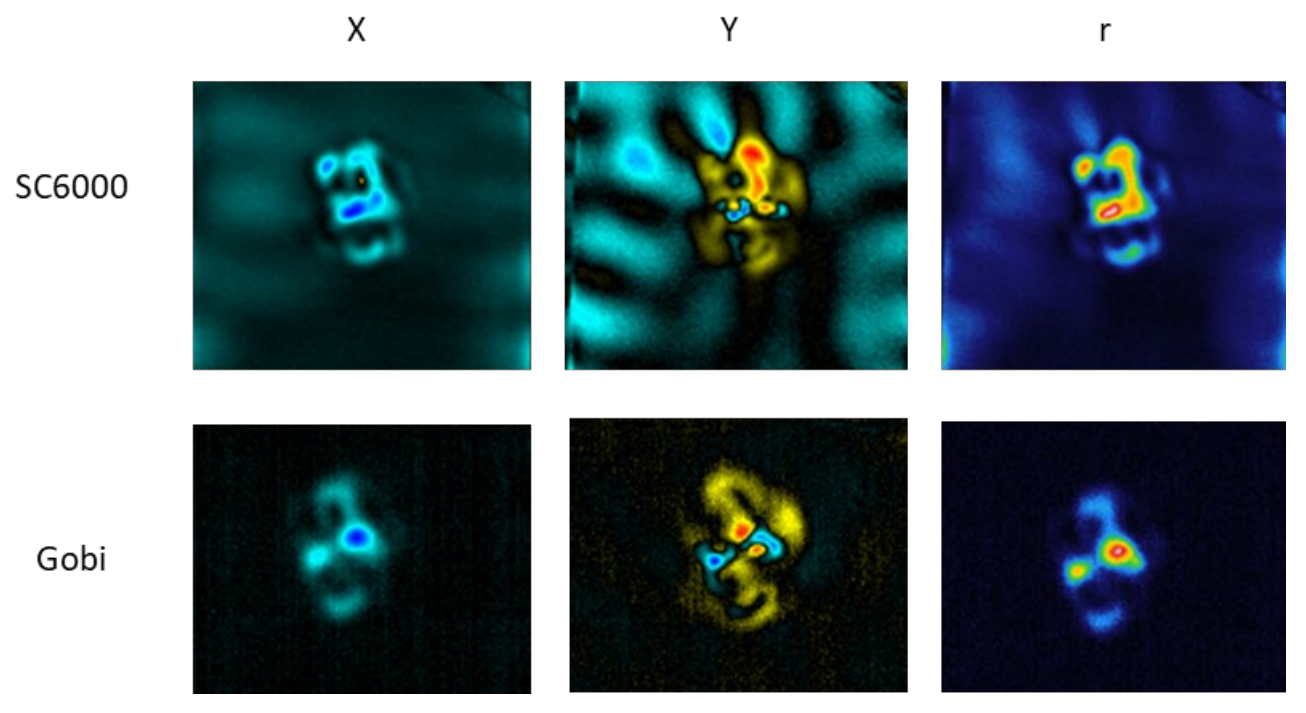

Figure 9. X, Y and r components measured on 21.44 J impact specimen, using a photon detector (top row) and microbolometer (bottom row).
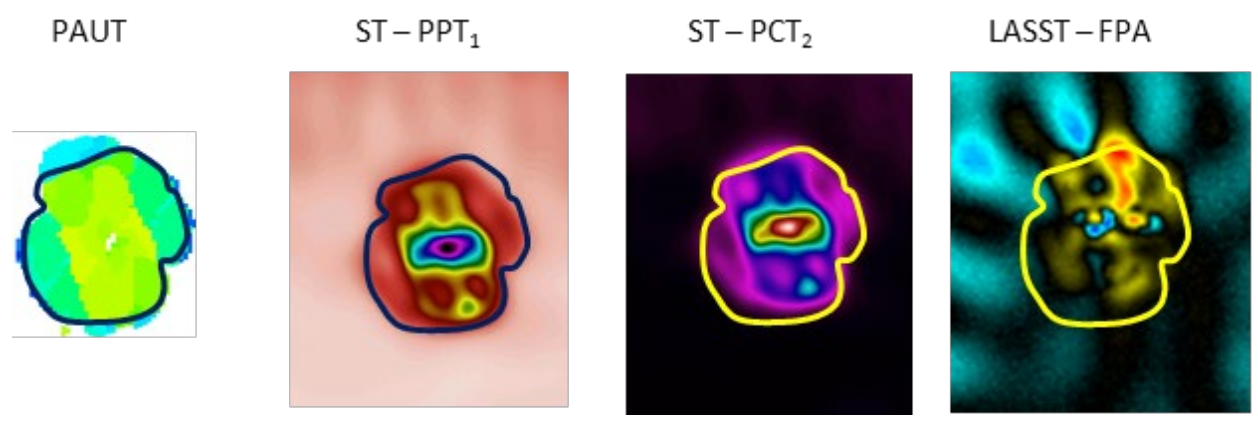

LASSTMicrobolometer

Figure 10. Comparison of PAUT, traditional ST (PPT $T_{1}$ and $\left.P C T_{3}\right)$ processed over $2 s$ and LASST (Y component) from the FPA and microbolometer for $21.44 \mathrm{~J}$ impact specimen.

Finally, a comparison between the results obtained from LASST, conventional ST and PAUT are shown in Fig. 10, with the outline being the traced damage area from the PAUT result corresponding to damage up to $1.8 \mathrm{~mm}$ in depth. The ST results correspond to an insonification time of $1 \mathrm{~s}$ with the response recorded over a $2 \mathrm{~s}$ observation time and then post processed using 
the PPT and PCT methods described in [7] and [8] respectively. Both show indications matching the area of the PAUT indication, as well as signal features which correlate with the different depths of damage. The LASST signatures are similar in area but have sharper signal features, some of which correlate with features in the PAUT scan. When combined with the corresponding X component results in the first column of Fig. 9, the LASST technique is seen to be effective for the detection and sizing of BVID in these panels. It should be noted that the LASST results required a pulsed insonification and observation time of $800 \mathrm{~s}$ in total.

\section{Conclusions}

The results from this study have indicated that LASST implemented using a low cost microbolometer is an effective means of identifying BVID and performs comparably to conventional ST implemented using a cooled photon detector. In some cases the extent of damage identified using LASST was smaller than that obtained from PAUT, which may be due to the relatively low excitation power being insufficient to generate frictional heating across the full extent of a defect. While LASST requires a longer observation time than traditional ST, it has two significant comparative advantages: it uses significantly less power so it reduces the risk of marring the surface of the structure under inspection and it enables use of a low-cost microbolometer which makes this technique much more portable, robust and affordable.

\section{References}

[1] K.A. Tsoi and N. Rajic, "Non-destructive evaluation of aircraft structural components and composite materials at DSTO using sonic thermography", DSTO Technical Note, DSTO-TN-0986 2011.

[2] K. Tsoi, C. Antolis and N. Rajic, "Penetration Depth Limits of Active Thermography for Detection of Impact Damage in Carbon Fibre Composites", 22nd International Conference on Composite Materials, 2019.

[3] N. Rajic and D. Rowlands, "Thermoelastic stress analysis with a compact low-cost microbolometer system”, Quantitative InfraRed Thermography Journal, 10 (2013) 135-158. https://doi.org/10.1080/17686733.2013.800688

[4] J. Rantala, D. Wu \& G. Busse, “Amplitude-Modulated Lock-In Vibrothermography for NDE of Polymers and Composites", Journal of Research in Nondestructive Evaluation, 7:4, (1996) 215-228. https://doi.org/10.1080/09349849609409580

[5] Salerno, A, Dillenz, A. Wu, D. Rantala J. and Busse, G., "Progress in ultrasound lockin thermography", Quantitative Infrared Thermography 4, (1998), 154-160. https://doi.org/10.21611/qirt.1998.024

[6] Zweschper, T, Riegert, G., Dillenz, A. and Busse, G., "Frequency-modulated elastic wave thermography," Proc. SPIE 5073, Thermosense XXV, 2003. https://doi.org/10.1117/12.488547

[7] X. Maldague and S. Marinetti, "Pulse Phase Infrared Thermography”, J. Appl. Phys., 79, (1996) pp. 2694-2698, 1996. https://doi.org/10.1063/1.362662

[8] N. Rajic. "Principal Component Thermography”. DSTO Technical Report DSTO-TR-1298, 2002. 